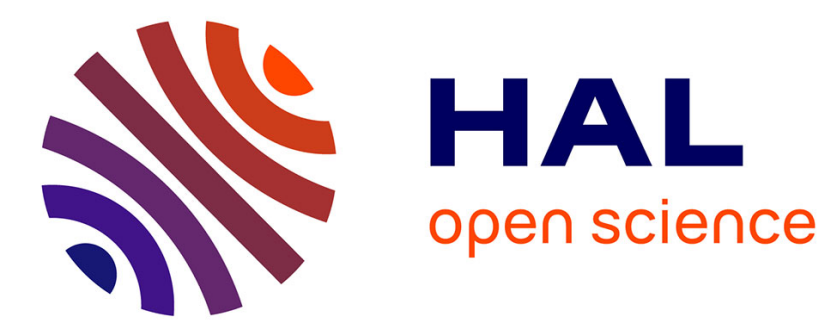

\title{
Robust Functional Interval Observer for Multivariable Linear Systems
}

\author{
Luc Meyer
}

\section{To cite this version:}

Luc Meyer. Robust Functional Interval Observer for Multivariable Linear Systems. Journal of Dynamic Systems, Measurement, and Control, 2019, 141 (9), 10.1115/1.4043334 . hal-02873793

\section{HAL Id: hal-02873793 \\ https://hal.science/hal-02873793}

Submitted on 18 Jun 2020

HAL is a multi-disciplinary open access archive for the deposit and dissemination of scientific research documents, whether they are published or not. The documents may come from teaching and research institutions in France or abroad, or from public or private research centers.
L'archive ouverte pluridisciplinaire HAL, est destinée au dépôt et à la diffusion de documents scientifiques de niveau recherche, publiés ou non, émanant des établissements d'enseignement et de recherche français ou étrangers, des laboratoires publics ou privés. 


\title{
Robust Functional Interval Observer for Multivariable Linear Systems
}

\author{
Luc Meyer \\ Research Engineer \\ Onera \\ Univ Paris Saclay \\ Palaiseau, France \\ Email: luc.meyer@onera.fr
}

The study of a continuous-time multivariable linear system may not need the knowledge of the entire internal state vector, but only of a linear function of it. In this case, instead of designing a complete observer, only a functional (also called reduced order) observer is used. In this field of research, this paper focuses on robust functional cooperative interval observers. Such an observer is proposed, and its properties (in particular, its convergence) are established. Then, a design procedure is given for practical use. Finally, the theoretical contributions are illustrated on examples.

\section{Introduction}

The knowledge of the internal state of a mechanical system has been a field of study for many years. Luenberger has proposed the first observers for deterministic multivariable linear systems [1] [2], the aim of an observer being to estimate the internal state of a system using only its inputs and outputs. Then functional observers have been widely used when only the knowledge of a linear function of the state is needed [3]. The use of such observers aims to design low order observer for estimation problem, and thus they are also called reduced order observers.

Let consider the following multivariable linear system:

$$
\left\{\begin{array}{ll}
\dot{x}(t) & =A x(t)+B u(t)+w(t) \\
y(t) & =C x(t)+v(t) \\
z(t) & =L x(t)
\end{array},\right.
$$

where $x \in \mathbb{R}^{n_{x}}$ is the state of the system, $u \in \mathbb{R}^{n_{u}}$ is the control input, $y \in \mathbb{R}^{n_{y}}$ is the output, and $z \in \mathbb{R}^{n_{z}}$ is the functional state (i.e. a linear function of the state). $A, B, C$ and $L$ are constant matrices with appropriate dimensions. Finally, $w \in \mathbb{R}^{n_{x}}$ and $v \in \mathbb{R}^{n_{y}}$ are disturbance vectors. It is assumed that $n_{z} \leq n_{x}$.

Luenberger observers have been widely derived for the treatment of such problems [4] [5] [6] [7] [8]. In particular [8] has given necessary and sufficient conditions for a class of functional observers to exist.
Apart from the Luenberger observers, another class of observers has been recently developed: the interval observers. Such observers are of particular interest for critical systems as they give at any time an upper and a lower bounds of the real state. Thus, they give a guaranteed set in which the system state belongs.

Two main approaches have been developed in that sense. In one hand, the algebraic approach uses forward/backward propagation with different kind of geometrical forms, as parallelotopes [9] [10] [11], ellipsoids [12] [13], or zoonotopes [14] [15]. In the other hand, the cooperative approach is based on Luenberger theory and aims to provide two Luenberger observers, one observer being an upper bound of the real state, the other observer being a lower bound of it.

Such observers have already been applied to several kind of systems: Linear Time Invariant (LTI) systems [16], Linear Time Variant (LTV) systems [17], Linear Parameter Variant (LPV) systems, with unknown inputs [18] [19], Lipschitz systems [20] [21], bilinear systems [22], or others specific classes of nonlinear systems [23].

However, to the best of the author knowledge, no functional observer has been developed for multivariable linear systems in the frame of interval observers.

The aim of the present paper is thus to propose a robust functional cooperative interval observer for linear systems such as (1).

The paper is divided as follow. In section 2 , some preliminaries used in the rest of the paper are provided. in section 3, the main result of the paper is given: under some conditions, the existence of such an observer is proved. Then, in section 4, a way of designing such an observer in practical application is given. Finally, in section 5, the use of the observer is illustrated on some examples.

\section{Preliminaries}

\subsection{Notations}

The set of real matrices with $n \times p$ elements is denoted by $\mathcal{M}_{n, p}(\mathbb{R})$. The subset of invertible matrices of $\mathcal{M}_{n, n}(\mathbb{R})$ is 

biguity) denotes the identity matrix of $\mathcal{M}_{n, n}(\mathbb{R})$, and $0_{n, p} \in$ $\mathcal{M}_{n, p}(\mathbb{R})$ (0 when there is no ambiguity) denotes the null matrix with $n$ rows and $p$ columns.

For any matrix $A \in \mathcal{M}_{n, p}(\mathbb{R}), A^{T}$ its transpose, and $A^{\dagger}$ its pseudo-inverse. For any square matrix $A \in \mathcal{M}_{n, n}(\mathbb{R}), \operatorname{tr}(A)$ denotes its trace, and $\operatorname{det}(A)$ its determinant. For any invertible matrix $A \in G L_{n}(\mathbb{R}), A^{-1}$ denotes its inverse.

The operators $\leq, \geq,<$ and $>$ are understood component wise for vectors and matrices.

If $A \in \mathcal{M}_{n, m}(\mathbb{R})$, let set $A^{+}=\max \{0, A\}, A^{-}=A-A^{+}$ (such that $A=A^{+}+A^{-}$with $A^{+} \geq 0$ and $A^{-} \leq 0$ ). $|A|=A^{+}-A^{-}$ is the element-wise absolute value of $A$.

\subsection{Useful definitions and notations}

In this subsection, some basic definitions and results on the interval observer theory are given. The following gives a definition of an interval observer for systems such as (1).

Definition 1. A couple of variable $(\bar{z}(t), \underline{z}(t))$ is an interval observer for the functional state $z(t)$ in (1) if and only if:

1. the following inequalities hold for any $t \geq 0$ :

$$
\underline{z}(t) \leq z(t) \leq \bar{z}(t)
$$

2. the total error $e_{z}(t)=\bar{z}(t)-\underline{z}(t)$ is asymptotically bounded:

$$
\exists M \geq 0, \quad \lim _{t \rightarrow \infty} e_{z}(t) \leq M .
$$

The following definition and lemmas concern the cooperative approach on which the cooperative interval observers are based.

Definition 2. A square matrix $A=\left(a_{i, j}\right) \in \mathcal{M}_{n}(\mathbb{R})$ is said to be Metzler if $a_{i, j} \geq 0$ for $i \neq j$.

Lemma 1. (Cooperative property, [24]) Let be a nonautonomous system described by $\dot{x}(t)=A x(t)+B(t)$ where $A$ is Metzler and $B(t) \geq 0, \forall t \geq 0$. Then, $x(t) \geq 0, \forall t \geq 0$, provided that $x(0) \geq 0$.

Lemma 2. [25] Given a system described by $\dot{x}(t)=A x(t)+$ $B(t)$ where $A$ is Metzlez and Hurwitz and $0 \leq B(t) \leq \bar{B}, \forall t \geq 0$. Then:

$$
\lim _{t \rightarrow \infty} x(t) \leq-A^{-1} \bar{B}
$$

providing that $x(0) \geq 0$.

Finally, let state two useful results for interval calculation.

Lemma 3 (Order Relation [26]). Let be a vector $x \in$ $[\underline{x} ; \bar{x}]$ and a matrix A with compatible dimensions (i.e. such that Ax has a sense). Then:

$$
A^{+} \underline{x}+A^{-\bar{x}} \leq A x \leq A^{+} \bar{x}+A^{-} \underline{x} .
$$

Lemma 4 (Extended Order Relation [26]). Let be a vector $x \in[\underline{x} ; \bar{x}]$ and a matrix $A \in[\underline{A} ; \bar{A}]$ with appropriate dimensions. Then:

$\underline{A}^{+} \underline{x}^{+}+\bar{A}^{+} \underline{x}^{-}+\underline{A}^{-} \bar{x}^{+}+\bar{A}^{-} \bar{x}^{-} \leq A x \leq \bar{A}^{+} \bar{x}^{+}+\underline{A}^{+} \bar{x}^{-}+\bar{A}^{-} \underline{x}^{+}+\underline{A}^{-} \underline{x}^{-}$.

\section{Main Result}

In this section, the main result of the paper is established. Let consider the following system

$$
\begin{cases}\dot{x}(t) & =\left(A+\Delta A_{\rho(t)}\right) x(t)+\left(B+\Delta B_{\rho(t)}\right) u(t)+w(t) \\ y(t) & =C x(t)+v(t) \\ z(t) & =L x(t)\end{cases}
$$

with the same notations as in (1), except for the matrices $\Delta A_{\rho(t)}$ and $\Delta B_{\rho(t)}$ that are parameter-varying matrices enabling to contain the uncertainties on the real values of $A$ and B. $\rho \in \Omega$ is an unknown parameter, taking its values inside the known and bounded set $\Omega \subset \mathbb{R}^{n_{\rho}}$.

System (7) can be rewritten as:

$$
\begin{cases}\dot{x}(t) & =A x(t)+B u(t)+\delta(t)+w(t) \\ y(t) & =C x(t)+v(t) \\ z(t) & =L x(t)\end{cases}
$$

where $\delta(t)=\Delta A_{\rho(t)} x(t)+\Delta B_{\rho(t)} u(t)$.

Note that $\rho$ being bounded, with known bounds, it is also the case of $\Delta A_{\rho(t)}$ and $\Delta B_{\rho(t)}$, i.e. there exists matrices $\overline{\Delta A}$, $\underline{\Delta A}, \overline{\Delta B}$ and $\underline{\Delta B}$ such that for all $\rho \in \Omega$ :

$$
\left\{\begin{array}{l}
\underline{\Delta A} \leq \Delta A_{\rho} \leq \underline{\Delta A} \\
\underline{\Delta B} \leq \Delta B_{\rho} \leq \underline{\Delta B}
\end{array} .\right.
$$

In order to state the main result, let us introduce some assumptions.

Assumption 1. There exist known functions $\bar{w}(t), \underline{w}(t) \in$ $\mathbb{R}^{n_{x}}, \bar{v}(t), \underline{v}(t) \in \mathbb{R}^{n_{y}}$, and $\bar{v}_{d}(t), \underline{v}_{d}(t) \in \mathbb{R}^{n_{y}}$ such that, for any $t \geq 0$ :

$$
\left\{\begin{array}{l}
\underline{w}(t) \leq w(t) \leq \bar{w}(t) \\
\underline{v}(t) \leq v(t) \leq \bar{v}(t) \\
\underline{v}_{d}(t) \leq \dot{v}(t) \leq \bar{v}_{d}(t)
\end{array} .\right.
$$

Moreover, there exist known constants $W \in \mathbb{R}^{n_{x}}, V \in \mathbb{R}^{n_{y}}$ and $V_{d} \in \mathbb{R}^{n_{y}}$, such that for any $t \geq 0$ :

$$
\left\{\begin{array}{l}
\bar{w}(t)-\underline{w}(t) \leq W \\
\bar{v}(t)-\underline{v}(t) \leq V \\
\bar{v}_{d}(t)-\underline{v}_{d}(t) \leq V_{d}
\end{array}\right.
$$



interval observer literature.

Assumption 2. The state $x(t)$ is bounded. More precisely, there exist known constants $\bar{X} \in \mathbb{R}^{n_{x}}$ and $\underline{X} \in \mathbb{R}^{n_{x}}$ such that:

$$
\underline{X} \leq x(t) \leq \bar{X}, \quad \forall t \geq 0 .
$$

Assumption 3. The input $u(t)$ is bounded. More precisely, there exist (not necessary known) constants $\bar{U} \in \mathbb{R}^{n_{u}}$ and $\underline{U} \in$ $\mathbb{R}^{n_{u}}$ such that:

$$
\underline{U} \leq u(t) \leq \bar{U}, \quad \forall t \geq 0
$$

Assumptions 2 and 3 are usually encountered in real cases, as in real physical systems, the state and the input have finite values, and thus are uniformly bounded. A discussion on these assumptions is given in remark 1 (after Theorem 7 proof).

Let set for all $t \leq 0: \bar{\delta}(t)=\underline{\Delta A}^{+} \underline{X}^{+}+\overline{\Delta A}^{+} \underline{X}^{-}+\underline{\Delta A^{-}} \bar{X}^{+}+$ $\overline{\Delta A}^{-} \bar{X}^{-}+\overline{\Delta B} u(t)^{-}+\underline{\Delta B} u(t)^{+}$and $\underline{\delta}(t)=\overline{\Delta A}^{+} \bar{X}^{+}+\overline{\Delta A}^{+} \bar{X}^{-}+$ $\overline{\Delta A}^{-} \underline{X}^{+}+\underline{\Delta A^{-}} \underline{X}^{-}+\underline{\Delta B} u(t)^{-}+\overline{\Delta B} u(t)^{+}$. Thus, under Assumption 2 , and using equations (9) and Lemma 4, it comes:

$$
\forall t \geq 0, \quad \underline{\delta}(t) \leq \delta(t) \leq \bar{\delta}(t)
$$

Now, let consider the following observer equations:

$$
\begin{cases}\dot{\bar{m}} & =U N U^{-1} \bar{m}+U J y+U H u+\bar{r}+\bar{s} \\ \dot{m} & =U N U^{-1} \underline{m}+U J y+U H u+\underline{r}+\underline{s} \\ \overline{\bar{z}} & =T^{+} \bar{m}+T^{-} \underline{m}+E y \\ \underline{z} & =T^{+} \underline{m}+T^{-} \bar{m}+E y\end{cases}
$$

where $U \in G L_{n_{x}}(\mathbb{R}), T=U^{-1}, N, J, H$ and $E$ are gain matrices to be determined, $\bar{r}$ and $\underline{r}$ are set to:

$$
\left\{\begin{aligned}
\bar{r}(t) & =[U(L-E C)]^{+} \bar{w}(t)+[U(L-E C)]^{-} \underline{w}(t) \\
& +[U(N E-J)]^{+} \bar{v}(t)+[U(N E-J)]^{-} \underline{v}(t) \\
& -[U E]^{+} \underline{v}_{d}(t)-[U E]^{-} \bar{v}_{d}(t) \\
\underline{r}(t) & =[U(L-E C)]^{+} \underline{w}(t)+[U(L-E C)]^{-} \bar{w}(t) \\
& +[U(N E-J)]^{+} \underline{v}(t)+[U(N E-J)]^{-} \bar{v}(t) \\
& -[U E]^{+} \bar{v}_{d}(t)-[U E]^{-} \underline{v}_{d}(t)
\end{aligned}\right.
$$

and finally, $\bar{s}$ and $\underline{s}(t)$ are set to:

$$
\left\{\begin{array}{l}
\bar{s}(t)=[U(L-E C)]^{+} \bar{\delta}(t)+[U(L-E C)]^{-} \underline{\delta}(t) \\
\underline{s}(t)=[U(L-E C)]^{+} \underline{\delta}(t)+[U(L-E C)]^{-} \bar{\delta}(t)
\end{array} .\right.
$$

Assumption 1 combined with Lemma 3 leads to the following result.
Lemma 5. For any $t \geq 0$, the following inequalities hold:

$$
\underline{r}(t) \leq U(L-E C) w(t)+U(N E-J) v(t)-U E \dot{v}(t) \leq \bar{r}(t) .
$$

Moreover, by setting:

$$
R=|U(L-E C)| W+|U(N E-J)| V-|U E| V_{d},
$$

it comes for any $t \geq 0$ :

$$
\bar{r}(t)-\underline{r}(t) \leq R
$$

Let state another useful result.

Lemma 6. For any $t \geq 0$, the following inequalities hold:

$$
\underline{s}(t) \leq U(L-E C) \delta(t) \leq \bar{s}(t)
$$

Moreover, there exists a constant $S$ such that, for any $t \geq 0$ :

$$
\bar{s}(t)-\underline{s}(t) \leq S .
$$

Proof. Using both equations (14) and (17) with Lemma 3 leads to the first assertion. Let prove the second one. The only time-dependency in $\bar{\delta}$ and $\underline{\delta}$ is with $u(t)$, but $u(t)$ is bounded according to Assumption 3, and thus $\bar{\delta}$ and $\underline{\delta}$ are uniformly bounded, i.e. there exist constants $\bar{\Delta}$ and $\underline{\Delta}$, such that for any $t \geq 0, \underline{\Delta} \leq \underline{\delta}(t)$ and $\bar{\delta}(t) \leq \bar{\Delta}$. Finally, by setting

$$
S=|U(L-E C)|(\bar{\Delta}-\underline{\Delta}),
$$

and using Lemma (3), the current Lemma is proved.

Let state the main result of the paper.

Theorem 7. Let assumptions 1, 2 and 3 hold.

$$
\text { Let set } \Sigma=\left[\begin{array}{c}
L \\
C \\
C A
\end{array}\right], F=L A \Sigma^{\dagger} \text {, and } G=I-\Sigma \Sigma^{\dagger} .
$$

Let assume that:

1. $\operatorname{rank}(\Sigma)=\operatorname{rank}\left(\left[\begin{array}{c}\Sigma \\ L A\end{array}\right]\right)$,

2. the couple $\left(F I_{N}, G I_{N}\right)$ is observable, (where $I_{N}=$ $\left[\begin{array}{ll}I_{n_{z}} & 0_{2 n_{y}, n_{z}}^{T}\end{array}\right]^{T}$ ).

In this case, there exits a matrix $Z$ of appropriate dimension, such that the matrix $F I_{N}+Z G I_{N}$ is Hurwitz, and such that the matrix $U$ can be chosen in a way that $U\left(F I_{N}+Z G I_{N}\right) U^{-1}$ is Metzler.

Then, provided that

$$
\underline{m}(0) \leq U z(0)-U E y(0) \leq \bar{m}(0),
$$




$$
\begin{cases}N & =(F+Z G) I_{N} \\ E & =(F+Z G) I_{E} \\ K & =(F+Z G) I_{K}, \\ J & =K+N E \\ H & =L B-E C B\end{cases}
$$

where $I_{E}=\left[\begin{array}{lll}0_{n_{z}, n_{y}}^{T} & I_{n_{y}} & 0_{n_{y}, n_{y}}^{T}\end{array}\right]^{T}$ and $I_{K}=\left[\begin{array}{lll}0_{n_{z}}^{T}+n_{y}, n_{y} & I_{n_{y}}\end{array}\right]^{T}$.

Proof. First of all, let us note that if the pair $\left(F I_{N}, G I_{N}\right)$ is observable, it is well known that $Z$ can be chosen such that all the poles of $F I_{N}+Z G I_{N}$ are negative and distinct. The poles being negative, it makes the former matrix an Hurwitz one. The poles being distinct, a Jordan transformation can be used to construct a matrix $U \in G L_{n_{z}}(\mathbb{R})$ such that $U\left(F I_{N}+\right.$ $\left.Z G I_{N}\right) U^{-1}$ is Metzler [27] [28].

Now, let us make the following change of variable:

$$
\xi(t)=U z(t), \quad \forall t \geq 0
$$

In particular, it comes that $\xi(t)=U L x(t)$, for any $t \geq 0$.

Then, let us introduce the variables $\bar{\xi}$ and $\underline{\xi}$ defined by:

$$
\left\{\begin{array}{l}
\bar{\xi}(t)=\bar{m}(t)+U E y(t) \\
\underline{\xi}(t)=\underline{m}(t)+U E y(t)
\end{array}\right.
$$

Now, we let us prove that $(\bar{\xi}, \xi)$ is an interval observer for $\xi$. Once it will be done, we will come back to the initial system (with $z, \bar{z}$ and $\underline{z}$ ) and show that the interval observer properties remain true. This way of doing enables us to work in a framework in which the state transition matrix of the error is Metzler, and thus to apply Lemma 1.

The upper interval error on $\xi$ is:

$$
\begin{aligned}
& \bar{e}_{\xi}(t) \\
& =\bar{\xi}-\xi \\
& =\bar{m}+U E y-U L x \\
& =\bar{m}+U(E C-L) L x+U E v
\end{aligned}
$$

Then, the dynamic of the error is:

$$
\begin{aligned}
& \dot{\bar{e}}_{\xi} \\
& =\dot{\bar{m}}+U(E C-L) \dot{x}+U E \dot{v} \\
& =U N U^{-1} \bar{m}+U J y+U H u+\bar{r}+\bar{s}+U(E C-L) A x \\
& +U(E C-L) B u+U(E C-L) \delta+U(E C-L) w+U E \dot{v} \\
& =U N U^{-1} \bar{m}+U J C x+U J v+U(E C-L) A x+\bar{r}+\bar{s} \\
& +U(H+E C B-L B) u+U(E C-L) \delta+U(E C-L) w+U E \dot{v} \\
& =U N U^{-1} \bar{\xi}-U N E C x-U N E v-U N U^{-1} \xi+U N U^{-1} U L x \\
& +U J C x+U(E C-L) A x+U(H+E C B-L B) u \\
& +\bar{r}+U J v+U(E C-L) w+U E \dot{v}+\bar{s}+U(E C-L) \delta \\
& =U N U^{-1} \bar{e}_{\xi}+U(-N E C+N L+E C A+J C-L A) x \\
& +U(H+E C B-L B) u+\bar{r} \\
& -[U(N E-J) v+U(L-E C) w-U E \dot{v}]+\bar{s}-U(L-E C) \delta .
\end{aligned}
$$

In the same way, we find the following dynamic for the lower interval error:

$$
\begin{aligned}
& \dot{e}_{\xi}=U N U^{-1} \underline{e}_{\xi}-U(-N E C+N L+E C A+J C-L A) x \\
& -U(H+E C B-L B) u+[U(N E-J) v+U(L-E C) w-U E \dot{v}] \\
& -\underline{r}+U(L-E C) \delta-\underline{s} .
\end{aligned}
$$

The matrix $U$ being non singular, the observer is an asymptotically bounded interval one if and only if:

1. $H=L B-E C B$,

2. $-N E C+N L+E C A+J C=L A$,

3. $\bar{e}_{\xi} \geq 0$, and $\underline{e}_{\xi} \geq 0$,

4. $\lim _{t \rightarrow \infty} \bar{e}_{\xi}+\underline{e}_{\xi}$ is bounded.

The first assertion is true according to theorem assumptions. Let focus on the second assertion. The unknown matrices are $N, J$ and $E$. By making the following change of variable (also used in [8])

$$
K=J-N E
$$

the new equation becomes

$$
N L+E C A+K C=L A,
$$

with the unknown matrices $N, K$ and $E$, which is equivalent to

$$
\left[\begin{array}{lll}
N & E & K
\end{array}\right] \Sigma=L A,
$$

which has a solution for $[N E K]$ if and only if the following rank condition holds:

$$
\operatorname{rank}(\Sigma)=\operatorname{rank}\left(\left[\begin{array}{c}
\Sigma \\
L A
\end{array}\right]\right)
$$

which is true according to the theorem assumptions. In this case the solutions of equation (33) are under the form

$$
[N E K]=F+Z G
$$


Copyright (chere $Z$ is any arbitrary matrix of appropriate dimension.

The value of $Z$ is chosen so that $N=F I_{N}+Z G I_{N}$ is Hurwtiz (see the first paragraph of the proof). Then, the other gains are given by:

$$
\begin{cases}K & =(F+Z G) I_{K} \\ E & =(F+Z G) I_{E}\end{cases}
$$

And finally, $J$ is easily obtained by $J=K+N E$.

Thus, using the gain matrices previously calculated, the interval error bounds are:

$$
\left\{\begin{aligned}
\dot{\bar{e}}_{\xi} & =U N U^{-1} \bar{e}_{\xi}+\bar{r}-[U(N E-J) v+U(L-E C) w-U E \dot{v}] \\
& +\bar{s}-U(L-E C) \delta \\
\dot{e}_{\xi} & =U N U^{-1} \underline{e}_{\xi}+[U(N E-J) v+U(L-E C) w-U E \dot{v}]-\underline{r} \\
& +U(L-E C) \delta-\underline{s}
\end{aligned}\right.
$$

where $N$ is Hurwitz and $U N U^{-1}$ is Metzler. Thus, using equations Lemma 5 and Lemma 6, it comes for any $t \geq 0$ :

$$
\left\{\begin{array}{l}
\bar{r}-[U(N E-J) v+U(L-E C) w-U E \dot{v}]+\bar{s}-U(L-E C) \delta \geq 0 \\
{[U(N E-J) v+U(L-E C) w-U E \dot{v}]-\underline{r}+U(L-E C) \delta-\underline{s} \geq 0}
\end{array}\right.
$$

Then, given that $\xi(0) \leq \xi(0) \leq \bar{\xi}(0)$ (which comes from equation (24) combined with equations (27) and (26)), Lemma 1 can be applied, and it comes that $\bar{e}_{\xi}(t) \geq 0$ and $\underline{e}_{\xi}(t) \geq 0$ for any $t \geq 0$, and thus:

$$
\underline{\xi}(t) \leq \xi(t) \leq \bar{\xi}(t), \quad \forall t \geq 0
$$

Moreover the total error $e_{\xi}=\bar{\xi}(t)-\underline{\xi}(t)$ satisfies:

$$
\dot{e}_{\xi}=U N U^{-1} e_{\xi}+(\bar{r}-\underline{r})+(\bar{s}-\underline{s})
$$

Then, recalling that $\bar{r}-\underline{r} \leq R$ (from Lemma 5), and $\bar{s}-\underline{s} \leq S$ (from Lemma 6), noting that $U N U^{-1}$ is Hurwitz (indeed, $N$ is Hurwitz and $U$ is non singular, and thus the matrices $N$ and $U N U^{-1}$ have the same eigenvalues), and using lemma 2, it comes that:

$$
\lim _{t \rightarrow \infty} e_{\xi} \leq-U N^{-1} U^{-1}(R+S)
$$

Finally, let us construct the interval observer of the initial system (7). Using (15) and (27), it comes:

$$
\left\{\begin{array}{l}
\bar{z}(t)=T^{+} \bar{\xi}(t)+T^{-} \underline{\xi}(t) \\
\underline{z}(t)=T^{+} \underline{\xi}(t)+T^{-} \overline{\bar{\xi}}(t)
\end{array}\right.
$$

Using Lemma 3 combined with equations (39) and (42), it follows for all $t \geq 0$ :

$$
\underline{z}=T^{+} \underline{\xi}+T^{-\bar{\xi}} \leq T \xi \leq T^{+} \bar{\xi}+T^{-} \underline{\xi}=\bar{z}
$$

and recalling that $z=T \xi$, it comes:

$$
\underline{z}(t) \leq z(t) \leq \bar{z}(t), \quad \forall t \geq 0
$$

Finally, the total error is given by

$$
e_{z}=\bar{z}-\underline{z}=T e_{\xi} \leq|T| e_{\xi}
$$

the last inequality coming from the fact that $e_{\xi} \geq 0$. Using (41), it follows that $\lim _{t \rightarrow \infty} e_{z}(t) \leq-|T| U N^{-1} U^{-1}(R+S)$, which makes the couple $(\bar{z}(t), \underline{z}(t))$ an asymptotically bounded interval observer and conclüdes the proof.

If there is no uncertainty on on system (7) matrices, nor perturbations on its equations, a stronger result can be stated.

Corollary 8. Let assume that there is no uncertainty on system (7) matrices $\left(\Delta A_{\rho(t)}=0\right.$ and $\Delta B_{\rho(t)}=0$, for any $\left.t \geq 0\right)$, and no perturbation $(w(t)=0$, and $v(t)=0$ for any $t \geq 0)$. Then, under the same conditions as in Theorem 7 (except for Assumptions 1, 2 and 3 that are not needed anymore), the conclusions of Theorem 7 remain true, and moreover:

$$
\lim _{t \rightarrow \infty} e_{z}(t)=0
$$

Proof. Note that without any uncertainty on system matrices nor perturbation on it, the proof of Theorem 7 remains correct if we set $R=S=0$ (which is coherent with the absence of uncertainty and perturbation), and thus the statements of this Theorem remains true.

In order to prove that $\lim _{t \rightarrow \infty} e_{z}(t)=0$, let consider the assertion given in the last paragraph of Theorem 7 proof: $\lim _{t \rightarrow \infty} e_{z}(t) \leq-|T| U N^{-1} U^{-1}(R+S)$. In our case, $R=S=0$, and thus $\lim _{t \rightarrow \infty} e_{z}(t)=0$, which concludes the proof.

Remark 1. Let us say some words about assumptions 2 and 3. As it has been said before, these two assumptions are not very restrictive as in real systems they always hold (physical parameters are always bounded).

Moreover, let us notice that in the case the matrices $A$ and $B$ are perfectly known, and are not parameter-varying, $\delta(t)=0$ for all $t \geq 0$, Theorem 7 holds without these two assumptions. Indeed, these assumptions are only used in order to guarantee bounds on $\delta(t)$.

Let us notice also that the bounds of the input do not need to be known in order to make the observer work (Assumption 3 does not state the knowledge of the bounds, but only the fact, that $u(t)$ has to be bounded). For the state, on the contrary, the maximum bound has to be known. Indeed, both $x(t)$ and $u(t)$ do appear in $\delta(t)$, but contrary to $u(t)$ which is known at each time step, it is obviously not the case of $x(t)$.

As a conclusion of this remark, the only restriction of the proposed observer dealing with uncertain matrices, holds in the requirement of the knowledge of the bounds of the state (which is also a requirement in others work dealing with uncertain systems [26] [29]). 


\section{Accepted manuscript posted April 1,2019. doi:10.1115/1.4043334}

Copyright Remark 2. Let us compare the proposed interval observer with classical Luenberger functional observers [7] [8]. The interval aspect developed in the present paper makes the estimation more robust in the sense that the knowledge of the couple $(\bar{z}(t), z(t))$ ensures that the true state belongs inside the given interval. On the contrary, with classical Luenberger observers, and even if the asymptotic convergence is stated, no upper bound of the effective distance between the true state and the estimated state is given (particularly on the beginning of the system simulation), and for some applications, this aspect can be very critical.

However, one drawback of the use of such an interval observer is the calculation time that can be a bit higher than for a classical Luenberger observer, because two observers have to be calculated (the upper and the lower ones).

Another drawback could be raised: the knowledge of the initial bounds, i.e. an interval in which the initial state (or the initial functional state) belongs. However, in real system, it is always possible to take arbitrary large initial bounds to avoid such an issue, but it leads to very pessimistic initial estimation (this is reduced over time thanks to the asymptotic bounds given by Theorem 7). Note that this issue is also true for classical Luenberger observer for which an initial guess is also needed for the observer initialization.

\section{Practical Design}

In order to use the proposed observer in practical use, the following design procedure can be applied.

1. Calculate $\Sigma=\left[\begin{array}{c}L \\ C \\ C A\end{array}\right], F=L A \Sigma^{\dagger}$, and $G=I-\Sigma \Sigma^{\dagger}$.

2. Calculate the projection matrices $I_{N}=\left[\begin{array}{ll}I_{n_{z}} & 0_{2 n_{y}, n_{z}}^{T}\end{array}\right]^{T}$, $I_{K}=\left[\begin{array}{lll}0_{n_{z}, n_{y}}^{T} & I_{n_{y}} & 0_{n_{y}, n_{y}}^{T}\end{array}\right]^{T}$ and $I_{K}=\left[\begin{array}{ll}0_{n_{z}+n_{y}, n_{y}}^{T} & I_{n_{y}}\end{array}\right]^{T}$.

3. Check the rank condition 1 and the observability condition 2 of Theorem 7.

4. Calculate the value of $Z$ using a classical pole placement (such that the poles of $F I_{N}+Z G I_{N}$ are all different and with negative real parts).

5. Construct a matrix $U \in G L_{n_{z}}(\mathbb{R})$ such that the matrix $U\left(F I_{N}+Z G I_{N}\right) U^{-1}$ is Metzler. This is always possible using a classical Jordan trnasformation (more on this can be found in [28] [27]).

6. Calculate $T=U^{-1}$, and calculate the observer gain matrices using (25).

7. By the knowledge of an interval $(\underline{x}(0), \bar{x}(0))$ of $x(0)$, calculate an interval for $z(0)$ (the following expressions being established using Lemma 3)

$$
\left\{\begin{array}{l}
\bar{z}(0)=L^{+} \bar{x}(0)+L^{-} \underline{x}(0) \\
\underline{z}(0)=L^{+} \underline{x}(0)+L^{-} \bar{x}(0)
\end{array},\right.
$$

Then, calculate $\underline{m}(0)$ and $\bar{m}(0)$ so that equation (24) holds, using the following expressions (expressions es- tablished with equation (24) and Lemma 3):

$$
\left\{\begin{array}{l}
\bar{m}(0)=U^{+} \bar{z}(0)+U^{-} \underline{z}(0)-U E y(0) \\
\underline{m}(0)=U^{+} \underline{z}(0)+U^{-} \bar{z}(0)-U E y(0)
\end{array} .\right.
$$

8. Equations (15) (used with equations (16) and (17)) give the upper and lower bounds of $z(t)$ for $t \geq 0$.

\section{Illustrative examples}

\subsection{Example 1}

Let consider the following example (adapted from [8]):

$$
\left\{\begin{aligned}
\dot{x}(t) & =\left[\begin{array}{ccc}
-2 & 1 & 0 \\
1 & -3 & 1 \\
0 & 0 & -1
\end{array}\right] x(t)+\left[\begin{array}{c}
1 \\
0 \\
-1
\end{array}\right] u(t) \\
y(t) & =\left[\begin{array}{lll}
1 & 0 & 0 \\
0 & 1 & 0
\end{array}\right] x(t) \\
z(t) & =\left[\begin{array}{lll}
1 & 1 & 0
\end{array}\right] x(t)
\end{aligned}\right.
$$

where each initial state is chosen randomly in $[-1 ; 1]$, and the input is set to $u(t)=0.4 \cos (1.5 t)$.

By applying the design procedure proposed in section 4 , we find the interval estimation given on Figure 1.

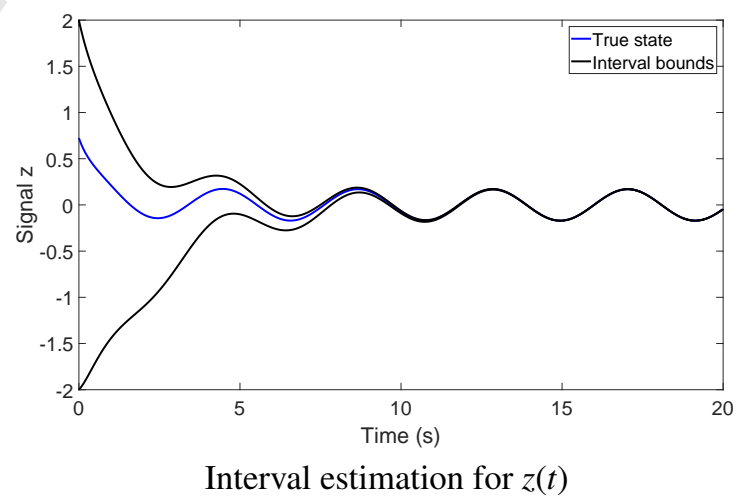

Fig. 1: Example 1. Interval estimation.

As stated in Corollary 8 (in the case in which there is no uncertainty on system matrices nor perturbation), the total error converges asymptotically towards 0 , which can be seen on Figure 1 by the fact that the two interval bounds get closer together, until they are mixed up.

\subsection{Example 2}

Let us consider a more complex case in which the input, as well as the signal of interest $z(t)$ are of dimension 2, and 

surement equations:

$$
\left\{\begin{array}{l}
\dot{x}(t)=\left[\begin{array}{ccc}
-2 & 1 & 0 \\
1 & -3 & 1 \\
0 & 0 & -1
\end{array}\right] x(t)+\left[\begin{array}{cc}
1 & 0 \\
0 & 1 \\
-1 & 0
\end{array}\right] u(t)+w(t) \\
y(t)=\left[\begin{array}{lll}
1 & 0 & 0 \\
0 & 1 & 0
\end{array}\right] x(t)+v(t) \\
z(t)=\left[\begin{array}{lll}
1 & 1 & 2 \\
0 & 0 & 1
\end{array}\right] x(t)
\end{array}\right.
$$

where each initial state is chosen randomly in $[-20 ; 20]$, the input is set to $u(t)=[4 \cos (1.5 t) 5 \sin (t)]^{T}$, the state perturbation is set to $w(t)=0.5[1-2 \operatorname{rand}(t) \cos (t)-\sin (t)]^{T}$ (where $\operatorname{rand}(t)$ gives an uniformly random number in $[0 ; 1])$, and the measurement perturbation is set to $v(t)=$ $0.1[\sin (0.7 t) \cos (-0.4 t)]^{T}$.

The known bounds of the perturbations (the perturbations $w(t)$ and $v(t)$ are unknown and unmeasurable) are $\bar{w}(t)=$ $0.5\left[\begin{array}{lll}1 & 1 & 1\end{array}\right]^{T}, \underline{w}(t)=-\bar{w}(t), \bar{v}(t)=0.1\left[\begin{array}{ll}1 & 1\end{array}\right]^{T}, \underline{v}(t)=-\bar{v}(t)$, $\bar{v}_{d}(t)=0.1\left[\begin{array}{ll}0.7 & 0.4\end{array}\right]^{T}$ and $\underline{v}_{d}(t)=-\bar{v}_{d}(t)$.

By applying the design procedure proposed in section 4 , we find the interval estimation given on Figures 2.

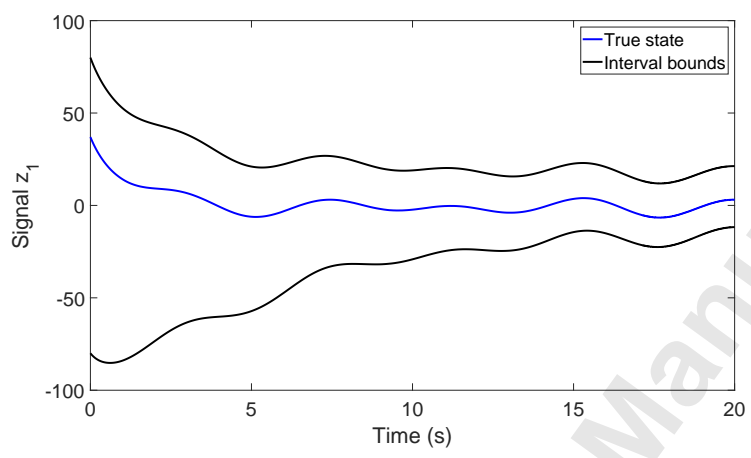

Interval estimation for $z_{1}(t)$

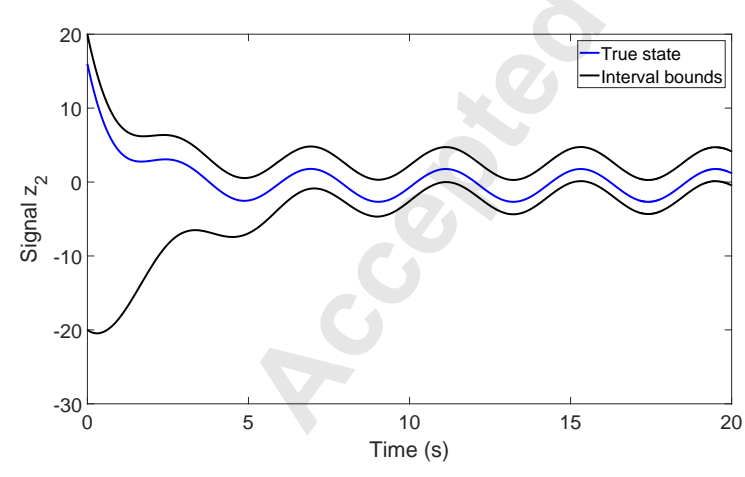

Interval estimation for $z_{2}(t)$

Fig. 2: Example 2. Interval estimation.

In this case, the presence of perturbations prevent from

\subsection{Example 3}

In this third example, let us consider the following system in which there are uncertainties on matrices system and perturbations in both the state and the measurement equa- 


$$
\begin{aligned}
\dot{x}(t)= & {\left[\begin{array}{ccc}
-2+0.15 \rho_{A} & 1 & 0 \\
1 & -3-0.05 \rho_{A} & 1 \\
0 & 0 & -1+0.45 \rho_{A}
\end{array}\right] x(t) } \\
& +\left[\begin{array}{cc}
1+0.14 \rho_{B} & 0.01 \rho_{B} \\
0 & 1+0.11 \rho_{B} \\
-1 & -0.07 \rho_{B}
\end{array}\right] u(t)+w(t) \\
y(t)= & {\left[\begin{array}{lll}
1 & 0 & 0 \\
0 & 1 & 0
\end{array}\right] x(t)+v(t) } \\
z(t)= & {\left[\begin{array}{lll}
1 & 1 & 0
\end{array}\right] x(t) }
\end{aligned}
$$

where each initial state is chosen randomly in $[-20 ; 20]$, the input is set to $u(t)=[4 \cos (1.5 t) 5 \sin (t)]^{T}$, the state perturbation is set to $w(t)=0.5[1-2 \operatorname{rand}(t) \cos (t)-\sin (t)]^{T}$, and the measurement perturbation is set to $v(t)=$ $0.1[\sin (0.7 t) \cos (-0.4 t)]^{T}$. Finally, the parameter is set to $\rho(t)=\left[\rho_{A}(t) \rho_{B}(t)\right]^{T}=[\cos (t) \sin (t)]^{T} \in \Omega$, where $\Omega=$ $[-1 ; 1] \times[-1 ; 1]$. Note that the evolution of the parameter $\theta$ is totally unknown from the user. However, the bounds of $\Omega$ are known.

It appears that $\Delta A(t)=\rho_{A}(t)\left[\begin{array}{ccc}0.15 & 0 & 0 \\ 0 & -0.05 & 0 \\ 0 & 0 & 0.45\end{array}\right]$ and $\Delta B(t)=\rho_{B}(t)\left[\begin{array}{cc}0.14 & 0.01 \\ 0 & 0.11 \\ 0 & -0.07\end{array}\right]$, so that one can set $\overline{\Delta A}=$ $\left[\begin{array}{ccc}0.15 & 0 & 0 \\ 0 & 0.05 & 0 \\ 0 & 0 & 0.45\end{array}\right], \underline{\Delta A}=-\overline{\Delta A}, \overline{\Delta B}=\left[\begin{array}{cc}0.14 & 0.01 \\ 0 & 0.11 \\ 0 & 0.07\end{array}\right]$ and $\underline{\Delta B}=$ $-\overline{\Delta B}$.

The known bounds of the perturbations are the same as in Example 2.

It is assumed that for any $t \geq 0, x(t) \in[-20 ; 20]^{3}$, so that Assumption 2 holds for $\bar{X}=\left[\begin{array}{lll}20 & 20 & 20\end{array}\right]$, and $\underline{X}=-\bar{X}$. Moreover, the input $u(t)$ is clearly bounded as it is a continuous signal on a finite time duration, and thus Assumption 3 also holds.

The other assumptions of Theorem 7 has checked, and by applying the design procedure proposed in section 4 , we find the interval estimation given on Figure 4.

\section{Conclusions}

This paper has been devoted to the construction of a robust functional interval observer for multivariable linear systems. Conditions for such an observer to exist, as well as proof of convergence are given. Besides, a design procedure for practical use is detailed. Finally, the theoretical contributions are illustrated on three examples.

\section{References}

[1] Luenberger, D. G., 1964. "Observing the state of a linear system”. IEEE transactions on military electronics,

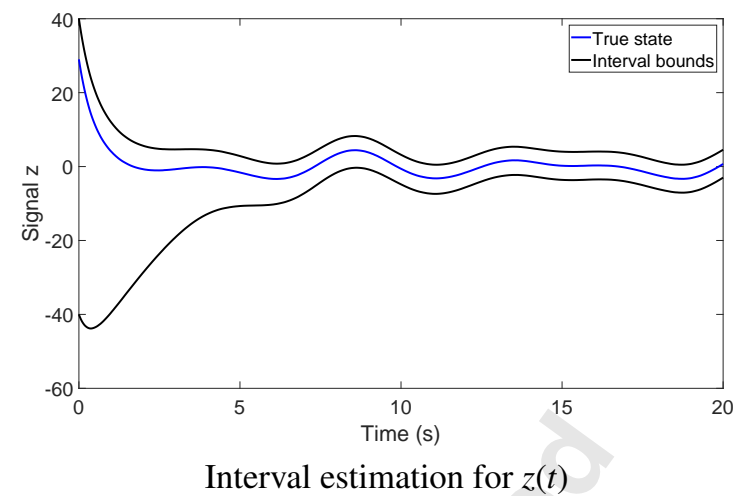

Fig. 4: Example 3. Interval estimation.

8(2), pp. 74-80.

[2] Luenberger, D., 1971. "An introduction to observers". IEEE Transactions on Automatic Control, 16, pp. 596602.

[3] Trinh, H., and Fernando, T., 2011. Functional observers for dynamical systems, Vol. 420. Springer Science \& Business Media.

[4] Tsui, C.-C., 1985. "A new algorithm for the design of multifunctional observers". IEEE Transactions on Automatic Control, 30(1), pp. 89-93.

[5] Helmick, R. E., Nagpal, K. M., and Sims, C. S., 1987. "Reduced-order estimation part 1. filtering". International Journal of Control, 45(6), pp. 1867-1888.

[6] Helmick, R. E., Nagpal, K. M., and Sims, C. S., 1987. "Reduced-order estimation part 2. smoothing". International journal of control, 45(6), pp. 1889-1898.

[7] Trinh, H., and Ha, Q., 2000. "Design of linear functional observers for linear systems with unknown inputs". International Journal of Systems Science, 31(6), pp. 741-749.

[8] Darouach, M., 2000. "Existence and design of functional observers for linear systems". IEEE Transactions on Automatic Control, 45(5), pp. 940-943.

[9] Jaulin, L., and Walter, E., 1993. "Set inversion via interval analysis for nonlinear bounded-error estimation". Automatica, 29(4), pp. 1053-1064.

[10] Jaulin, L., Kieffer, M., Didrit, O., and Walter, E., 2001. Applied interval analysis: with examples in parameter and state estimation, robust control and robotics.

[11] Jaulin, L., 2002. "Nonlinear bounded-error state estimation of continuous time systems". Automatica, 38, pp. 1079-1082.

[12] Maksarov, D., and Norton, J., 1996. "State bounding with ellipsoidal set description of the uncertainty". International Journal of Control, 65(5), pp. 847-866.

[13] Liu, Y., Zhao, Y., and Wu, F., 2016. "Ellipsoidal statebounding-based set-membership estimation for linear system with unknown-but-bounded disturbances". IET Control Theory \& Applications, 10(4), pp. 431-442.

[14] Alamo, T., Bravo, J. M., and Camacho, E. F., 2005. "Guaranteed state estimation by zonotopes". Automat- 
[15] Wang, Y., Wang, Z., Puig, V., and Cembrano, G., 2018. "Zonotopic set-membership state estimation for discrete-time descriptor lpv systems". IEEE Transactions on Automatic Control.

[16] Efimov, D., Perruquetti, W., Raissi, T., and Zolghadri, A., 2013. "On interval observer design for timeinvariant discrete-time systems". In European Control Conference 2013.

[17] Efimov, D., Perruquetti, W., Raissi, T., and Zolghadri, A., 2013. "Interval observers for time-varying discretetime systems". IEEE Transactions on Automatic Conctrol.

[18] Mazenc, F., and Bernard, O., 2010. "Interval observers for linear systems with additive disturbances". IFAC Proceedings Volumes, 43(14), pp. 30-35.

[19] Meyer, L., Ichalal, D., and Vigneron, V., 2017. "Interval observer for lpv systems with unknown inputs". IET Control Theory $\mathcal{E}$ Applications.

[20] Moisan, M., and Bernard, O., 2010. "Robust interval observers for global lipschitz uncertain chaotic systems". Systems \& Control Letters, 59(11), pp. 687-694.

[21] Meyer, L., Ichalal, D., and Vigneron, V., 2018. "Interval observer for nonlinear lipschitz systems with unknown inputs". In 2018 Annual American Control Conference (ACC), IEEE, pp. 5962-5967.

[22] Meyer, L., Ichalal, D., and Vigneron, V., 2018. "Interval observer for bilinear systems with unknown inputs". In 2018 Annual American Control Conference (ACC), IEEE, pp. 5957-5961.

[23] Efimov, D., Raissi, T., Stanislav, C., and Zolghadri, A., 2013. "Interval state observer for nonlinear time varying systems". Automatica, 49, pp. 200-205.

[24] Smith, H., 1995. Monotone dynamical systems: An introduction to the theory of competitive and cooperative systems, Vol. 41 of Mathematical Surveys and Monographs. Providence, Thode Island.

[25] Gouze, J., Rapaport, A., and Hadj-Sadok, M., 2000. "Interval observers for uncertain biological systems". Ecological Modelling, 133(1?2), pp. 45 - 56.

[26] Efimov, D., Fridman, L., Raissi, T., Zolghadri, A., and Seydou, R., 2012. "Interval estimation for lpv systems applying high order sliding mode techniques". Automatica, 48(9), pp. 2365-2371.

[27] Raïssi, T., Efimov, D., and Zolghadri, A., 2012. "Interval state estimation for a class of nonlinear systems". IEEE Transactions on Automatic Control, 57(1), pp. 260-265.

[28] Mazenc, F., and Bernard, O., 2011. "Interval observers for linear time-invariant systems with disturbances". Automatica, 47(1), pp. 140 - 147.

[29] Chebotarev, S., Efimov, D., Raïssi, T., and Zolghadri, A., 2015. "Interval observers for continuous-time lpv systems with 11/12 performance". Automatica, 58, pp. 82-89. 